\title{
Norois
}

Environnement, aménagement, société

\section{Politique de l'eau et aménagement urbain La « Renaturation » de la rivière Saint-Charles à Québec}

Water policy and urban planning. "Renaturation" of the Saint-Charles River in Quebec

\section{Alexandre Brun}

\section{(2) OpenEdition}

\section{Journals}

Édition électronique

URL : http://journals.openedition.org/norois/3603

DOI : $10.4000 /$ norois.3603

ISSN : $1760-8546$

\section{Éditeur}

Presses universitaires de Rennes

Édition imprimée

Date de publication : 30 juin 2011

Pagination : 89-107

ISBN : 978-2-7535-1479-9

ISSN : 0029-182X

\section{Référence électronique}

Alexandre Brun, « Politique de l'eau et aménagement urbain La « Renaturation » de la rivière SaintCharles à Québec », Norois [En ligne], 219 | 2011, mis en ligne le 30 septembre 2013, consulté le 01 mai 2019. URL : http://journals.openedition.org/norois/3603 ; DOI : 10.4000/norois.3603

Ce document a été généré automatiquement le 1 mai 2019.

(c) Tous droits réservés 


\section{Politique de l'eau et aménagement urbain La « Renaturation » de la rivière Saint-Charles à Québec}

Water policy and urban planning. "Renaturation" of the Saint-Charles River in Quebec

\section{Alexandre Brun}

1 Du milieu des années 1990 à 2009, la Ville de Québec a assuré la maitrise d'ouvrage d'études et de travaux destinés à « renaturer ${ }^{1}$ » la section aval de la rivière Saint-Charles. La « renaturation » ne désigne pas tant un retour à un état considéré comme « naturel » du cours d'eau qu'une logique de restauration de ses fonctions naturelles. À Québec, l'opération s'est traduite par la démolition des berges de béton érigées à grands frais en 1970. Une fois démolies, les berges ont été talutées en pentes douces et généreusement plantées de sorte qu'elles présentent désormais un aspect relativement «naturel ». Des travaux d'assainissement ont, en parallèle, permis de collecter, de stocker et de traiter les eaux pluviales plus efficacement que par le passé. La reconstitution du continuum végétal et aquatique qui relie la basse-ville avec l'arrondissement de la Haute-Saint-Charles a conquis les riverains et les touristes : « la rivière renaît » résumait Le Devoir en 2008.

2 La « renaturation » de la rivière Saint-Charles a joué un rôle catalyseur au plan urbain et environnemental car elle a répondu de manière concomitante aux demandes des riverains (une rivière propre), aux exigences réglementaires de l'Administration provinciale (améliorer la gestion des eaux pluviales), aux aspirations des élus (ne plus figurer sur la liste des villes canadiennes où l'eau est très polluée) et aux stratégies de promoteurs immobiliers (en quête de terrains constructibles). Le contexte, toutefois, s'y est prêté avec la préparation du $400^{\mathrm{e}}$ anniversaire de la fondation de la ville de Québec qui allait en 2008 attirer des centaines de milliers de visiteurs canadiens et étrangers.

Cette expérience témoigne moins d'un changement de paradigme en matière de planification territoriale - l'étalement urbain reste, si l'on peut dire, «la règle » - que d'un réel opportunisme stratégique de la Ville. À long terme, l'urbanisation quasi 
anarchique du bassin versant de la rivière Saint-Charles constitue néanmoins une menace sérieuse sur la ressource en eau. C'est peut être au travers de cette problématique que la gouvernance municipale - perçue aujourd'hui comme le seul système de décision légitime aux yeux des aménageurs - se tournera davantage vers l'organisme de bassin de la SaintCharles mis en place dans le prolongement de la Politique nationale de l'eau du Québec de 2002.

4 Cet article s'inscrit dans le cadre du programme de recherche interdisciplinaire francoquébécois «IDEAUX » (Pour une Intégration des politiques de Développement, de l'Eau, d'Aménagement et d'Urbanisme en faveur des milieuX aquatiques) ayant pour objet l'étude du jeu d'influence mutuelle entre les politiques dites de "territoires » et les politiques d'aménagement et de gestion des eaux. Ce projet a été initié en 2008 par le bureau d'études Sogreah avec l'appui technique et scientifique de l'unité mixte de recherche "Art-Dev » en réponse à l'appel à projets de recherche «Eaux \& Territoires » lancé conjointement par le ministère français de l'Écologie, le Centre national de la recherche scientifique, le Cemagref ainsi que le ministère des Ressources Naturelles et de la Faune du Québec².

5 Le dispositif méthodologique, basé sur des enquêtes et des «terrains » en France comme au Québec, a surtout reposé sur l'examen des stratégies aménagistes et les logiques qui les sous-tendent. Il s'est agi d'interroger les acteurs de l'aménagement au sens large (promoteurs immobiliers, banques et assureurs, urbanistes, entreprises du bâtiment et des travaux publics, élus locaux, bureaux d'études techniques, services de l'État...); l'objectif étant d'appréhender les problèmes de l'eau (gestion des pénuries, prévention du risque inondation, pollutions urbaines...) en inversant le regard qui leur est porté, c'est-àdire en se plaçant du côté des acteurs de l'aménagement et non du côté des acteurs de l'eau.

6 Plusieurs interrogations de nature opérationnelle ont guidé la recherche, et, in fine, les entretiens ouverts: faut-il impliquer davantage les acteurs de l'aménagement dans la gouvernance de l'eau, et, si oui, lesquels en priorité et comment, alors que seuls la diversification et le durcissement des standards environnementaux contribuent à l'amélioration - toute relative - des pratiques des acteurs de l'aménagement (Brun, 2010) ? Réciproquement, pourquoi favoriser l'implication des acteurs de l'eau dans la conduite de projets d'aménagement (quels projets?) et dans quelles conditions compte tenu que le processus de "territorialisation» de l'eau ne s'est pas accompagné, à quelques exceptions près, de la mise en place d'une gouvernance associant réellement eau et territoires (Brun et Lasserre, 2006 ; Ghiotti, 2007) ?

7 «IDEAUX » s'est déroulé schématiquement en deux séquences consécutives menées en parallèle en France et au Canada en différents bassins où l'urbanisation du territoire pose des problèmes aux gestionnaires publics et privés de l'eau (protection des aires d'alimentation en eau potable et des zones humides rendues difficiles en raison des pressions urbaines, système d'assainissement sous-dimensionné par rapport aux besoins, vulnérabilité accrue des biens et des personnes au risque d'inondation, etc.). La première séquence, d'une durée de 18 mois, a été consacrée aux enquêtes auprès d'acteurs de l'aménagement dans le but de modéliser leurs stratégies; 90 entretiens individuels ouverts ont été réalisés dans ce cadre dont une vingtaine concernait la basse rivière

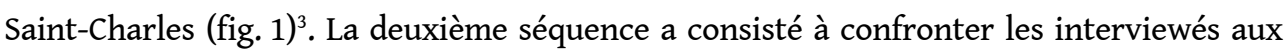
constats des chercheurs dans le cadre d'un débat relatif à la pérennité du système « eauterritoire ». Les entretiens confirment en particulier la faiblesse des instruments relatifs à 
la protection et à la gestion de l'eau par rapport aux logiques d'aménagement (excepté les aspects réglementaires quand ils sont appliqués). Or, paradoxalement, tant en France qu'au Québec, les aménageurs sont de plus en plus impliqués dans des démarches environnementales basées sur le volontariat (certification environnementale, labellisation d'écoquartiers, haute qualité environnementale des bâtiments, etc.).

Après un retour sur l'opération d'aménagement de la basse Saint-Charles menée dans les années 1960 et 1970, l'article présente le processus qui a présidé à la «renaturation » de cette section de la rivière et montre combien le rôle de la municipalité reste capital en dépit de la mise en place d'une gouvernance locale de l'eau au titre de la politique nationale de l'eau du Québec.

\section{La rivière Saint-Charles : un axe majeur du développement urbain de Québec}

La rivière Saint-Charles draine un bassin versant de $550 \mathrm{~km}^{2}$, qui compte parmi les plus anciennement colonisés et les plus densément peuplés du Québec (350 000 habitants). Son parcours d'environ $35 \mathrm{~km}$ entre sa source - le lac Saint-Charles - et son embouchure dans le Saint-Laurent traverse différentes formations géologiques - du bouclier canadien aux basses-terres du Saint-Laurent. Le cours de la rivière est divisible en trois segments distincts : de la source à la chute Kabir Kouba près de Wendake (première segment, faible pente, cours d'eau en milieu humide semi-forestier), de la chute jusqu'au Parc des Saules (deuxième segment, pente plus forte, cours d'eau encaissé à caractère torrentueux), et, enfin, du Parc des Saules à l'embouchure (troisième segment, faible pente en zone urbaine). C'est ce segment qui a constitué un axe majeur du développement de la ville (Lebel et Roy, 2000) et fait l'objet de la régénération actuelle.

Figure 1 : Carte de localisation du bassin versant de la rivière Saint-Charles, Service de cartographie, Université Laval (2010)/Location map : the watershed of the St. Charles River

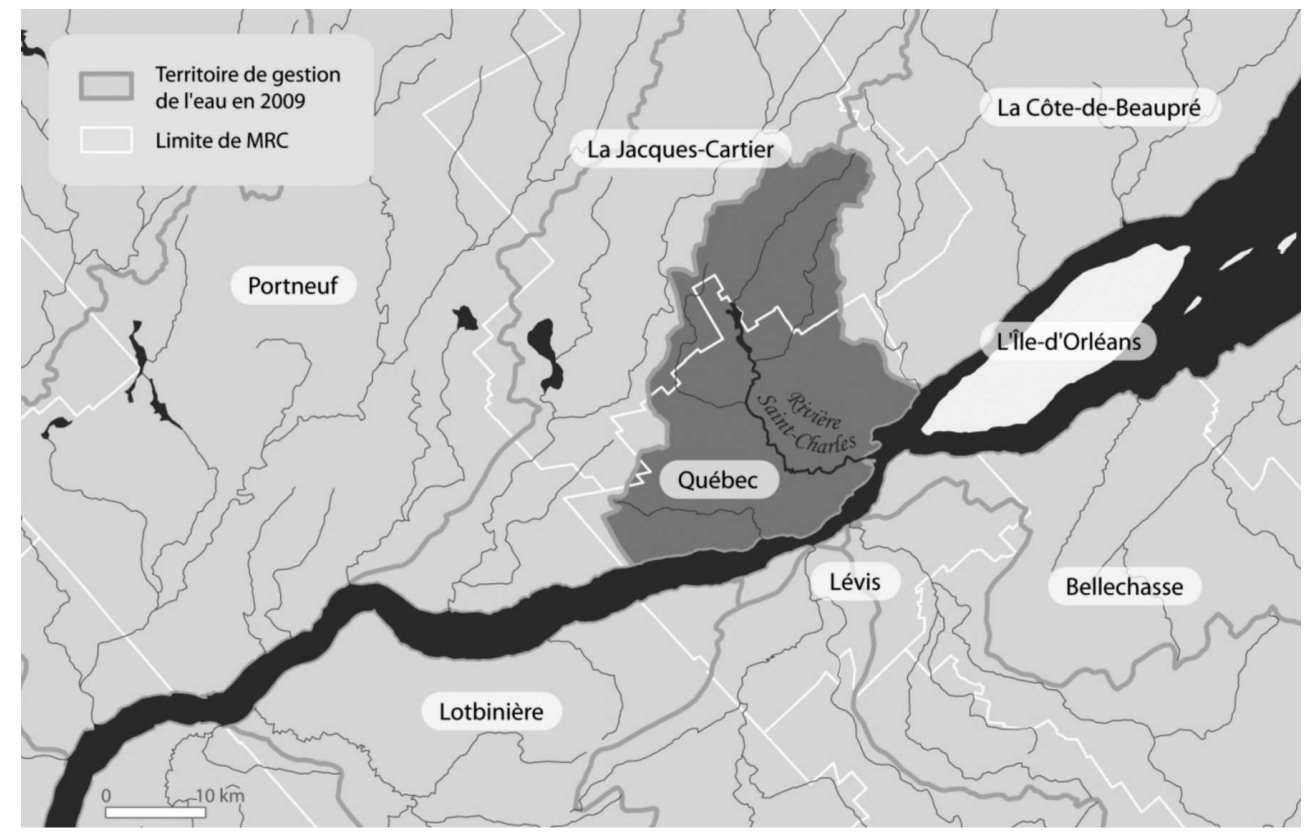




\section{Un site favorable au développement urbain et industrielle de la basse-ville}

10 «Québec est considérée comme la toute première colonie française en Amérique du Nord et le plus vieille établissement européen au nord du Rio Grande. Jacques Cartier y séjourne en 1535-1536. Samuel de Champlain fonde plus tard en 1608 la ville de Québec qui se développe, d'abord autour de l'Abitation (Place Royale), ensuite sur les berges de la rivière Saint-Charles » rappelle Y. Pouliot (2005). Dans sa partie aval, la rivière a fait l'objet d'aménagements successifs directement liés au développement de la basse ville de Québec. Dans la seconde moitié du XVII ${ }^{e}$ siècle, le lit de la rivière a été creusé pour garantir un tirant d'eau suffisant aux navires et les berges ont pour partie été remblayées suite à l'implantation des premiers chantiers navals. À la suite de l'indépendance américaine et du blocus continental napoléonien, l'empire britannique a choisi de sécuriser l'approvisionnement des ressources forestières de l'est du Canada et d'industrialiser la fabrication des bateaux à Québec. Entre 1820 et 1899, 1760 navires sont ainsi construits à Québec. Or, la majorité des chantiers maritimes sont localisés dans l'estuaire de la Saint-Charles. Au cours de cette période, la population du quartier SaintRoch a constamment augmenté passant de 829 habitants en 1795 à 10760 en 1842. Le flottage du bois et les effluents d'origine industrielle et domestique causent alors une dégradation de la qualité des eaux et une réduction de la diversité biologique.

11 À partir de 1870, les chantiers ont décliné car les navires d'acier propulsés par la vapeur (qui ont montré leur intérêt lors de la guerre de Sécession aux États-Unis entre 1861 et 1865) ont remplacé les bateaux à voiles en coque de bois : les manufactures (textile) se sont peu à peu substituées aux chantiers navals, alors même que l'attractivité de SaintRoch a été renforcée grâce à l'ouverture, en 1878, de la gare du Canadien Pacifique. Les nuisances olfactives et les épidémies attribuées à une mauvaise qualité de l'eau étaient telles que la municipalité a essayé de corriger la situation avec la construction, en 1852, de la première ligne d'aqueduc en provenance du lac Saint-Charles. Ce dernier, situé à la limite nord de la ville de Québec, a une superficie de $3,6 \mathrm{~km}^{2}$. Étant utilisé comme réservoir d'eau potable par la Ville de Québec, celle-ci a érigé un barrage à la sortie du lac en 1934 pour rehausser le niveau de l'eau: les basses terres avoisinantes ont été submergées sur des largeurs allant jusqu'à $50 \mathrm{~m}$. Cette mise en eau a donc bouleversé l'équilibre physique et biologique en modifiant la morphométrie du lac et en créant de manière périodique un déficit en oxygène important sur la moitié de la colonne d'eau. De nos jours, le niveau du lac est surélevé d'environ 2 mètres par rapport au niveau originel.

\section{0-2010 : du bétonnage à la « renaturation » de la basse Saint- Charles}

Dans un rapport alarmant, la Commission Surveyer a décrit en 1936 le cours d'eau comme l'un des plus pollués de la province (Chambre de commerce de Québec, 1962). Principal exutoire des eaux usées de l'agglomération et arrière-cour d'industries en déclin, la rivière Saint-Charles a de nouveau préoccupé la municipalité de Québec après-guerre. En 1966, à la suite d'une entente tripartite entre les gouvernements municipal, provincial et fédéral, il a été décidé que les berges seraient bétonnées (Dumont, 1998). Ainsi, après avoir mené à bien de vastes opérations de remblaiement (1957) destinées à accroître les 
surfaces constructibles, la municipalité de Québec a bétonné les berges de la rivière SaintCharles entre 1969 et 1974 dans le but de les rendre plus attrayantes. Elle a également assuré la construction d'une station d'épuration et optimisé la gestion des eaux urbaines afin de combler les insuffisances manifestes en matière d'assainissement dans les quartiers Saint-Roch et Limoilou en basse ville (Service de l'urbanisme de la Ville de Québec, 1973). Elle a enfin érigé un barrage anti-marée au début des années 1970 (le barrage Samson), de manière à stabiliser le niveau du plan d'eau en ville.

Ces travaux avaient pour objectif d'améliorer la gestion des eaux dans une perspective hygiéniste tout en modernisant la basse ville que traverse la rivière. L'époque - celle du «Plan Gréber Fiset ${ }^{4}$ » - était au fonctionnalisme. Entre le pont Scott à l'ouest de la Ville et la confluence avec le Saint-Laurent à l'Est, les infrastructures routières ont ainsi supplanté le corridor naturel de la Saint-Charles. Les stratégies d'aménagement urbain conduites à Québec faisaient écho aux « modèles » européens comme les voies sur berges à Paris. "Nous avons fini de rêver en couleurs, nous commençons maintenant des rêves de béton" déclarait alors Gilles Lamontagne, maire de Québec ${ }^{5}$. Grâce aux travaux effectués à la fin des "Trente Glorieuses ", la partie la plus urbaine de la rivière SaintCharles n'était plus le " cloaque charroyant déchets et débris, exposant un sol fangeux et empuanti » décrit par les J. Gréber et $\mathrm{E}$. Fiset en 1956. La qualité de l'eau restait cependant très médiocre à cause d'une gestion des eaux pluviales encore perfectible tandis que le marnage accentuait la perception des problèmes. Les aménagements n'avaient en outre pas rendu la rivière plus attrayante, au contraire. La municipalité de Québec s'est donc résolue à intervenir de nouveau, trente ans plus tard, en poursuivant les mêmes objectifs mais en adoptant une approche radicalement différente (fig. 2).

Un plan d'aménagement conçu en 1974 dénommé « Kabir-Kouba » (nom huron-wendat, qui signifie " rivière aux mille méandres ») ayant pour but de replacer la rivière au centre de la politique d'urbanisme de la Ville et auquel se sont greffés des objectifs écologiques a, dans cette perspective, été mis à jour en 1995 (Paulin, 2001). Du point de vue technique, le génie végétal a pour partie remplacé le génie civil : les huit kilomètres de berges de la rivière - bétonnés pour 16 millions de dollars canadiens quelques dizaines d'années plus tôt - ont été démolis et « renaturés » (Ville de Québec, 1996 ; Paulin, 2001). Une fois mise en scène, c'est l'eau qui "fait lien" entre les différentes opérations d'urbanisme (Scherrer, 2004), comme cela a été le cas auparavant à Portland, Chicago ou Ottawa (canal Rideau), mais dans une ambiance plus végétale.

L'opération a consisté à aménager des habitats fauniques diversifiés et à favoriser le redéveloppement de la ripisylve tout en garantissant la vocation récréative et l'accessibilité du cours d'eau à la population locale (Paulin, 2001; Beaulieu, 2006; Pronovost, 2009). Un réseau de sentiers pédestres de 32 kilomètres, fréquemment doublé de voies cyclables, relie désormais les secteurs restaurés au lac Saint-Charles (photos 1 et 2). La construction de douze bassins de rétention d'eau pluviale (ouvrages souterrains maçonnés) disposés près des émissaires sous les espaces verts relève d'un traitement plus classique. Elle a permis de réduire la fréquence des déversements dans le cours d'eau qui surviennent lors des précipitations pluvieuses, de 55 à 4 par an en captant environ 90 à $95 \%$ des volumes débordant des réseaux unitaires d'égouts en cas de pluie. Le coût global de l'opération s'est élevé à 115 millions de dollars environ, répartis à parts égales entre la municipalité de Québec, et les gouvernements provincial et fédéral. Les travaux de récupération, de stockage et d'évacuation des eaux pluviales vers les collecteurs 
principaux ont représenté une part très importante des dépenses publiques (plus de $80 \%$ ) .

16 Le projet urbain conduit par la Ville de Québec entre 1996 et 2009 au terme d'une série de consultations publiques a donc débouché sur des aménagements spectaculaires (Pronovost, 2009). Si le gain «social» reste difficilement quantifiable à ce stade, la présence de promoteurs de plus en plus nombreux sur les parcelles d'anciennes friches industrielles jouxtant le parc linéaire de la Saint-Charles témoigne d'une certaine forme de revitalisation urbaine; une basse ville mixte se dessine car l'habitat social des quartiers ouvriers sera complété par des lotissements plus cossus avec "vue sur la rivière » (Boutet, 2006). Cette opération a également intégré les habituels impératifs de sécurité publique et d'accessibilité pour les handicapés qui préoccupent les agglomérations. Une attention particulière a par exemple été portée à l'éclairage public et à la hauteur de la végétation, de sorte que le promeneur ait toujours un champ de vision éclairé et ouvert. Le mobilier urbain est adapté aux intempéries, aux inondations et aux dégradations volontaires. Les pentes des cheminements longeant la rivière sont suffisamment douces pour les personnes à mobilité réduite (Pronovost, 2009).

Le projet urbain a bénéficié d'une «fenêtre d'opportunités » qui s'est ouverte au cours des années 1990. Dans la perspective du $400^{\mathrm{e}}$ anniversaire de la fondation de la ville de Québec en 2008, le gouvernement Bouchard a en effet sélectionné, dès le printemps 2000, trois chantiers prioritaires, en tête desquels figurait la dépollution de la Saint-Charles. D'importants moyens ont donc été débloqués dans le but d'extraire la rivière de la liste noire des rivières les plus polluées d'Amérique du Nord car la capitale de la province francophone allait devenir la vitrine du Canada lors de festivités internationales ${ }^{6}$. Les fleuves sont ainsi régulièrement mis en scène lors de grands évènements dont l'approche précipite l'aménagement. La transformation des quais de la Tamise a par exemple connu deux soudaines accélérations à l'occasion des célébrations de l'an 2000 et des cinquante années du règne d'Elisabeth II (Lemonier, 2003). 
Figure 2 : Périmètre de « renaturation » de la basse rivière Saint-Charles à Québec/Renaturation zone

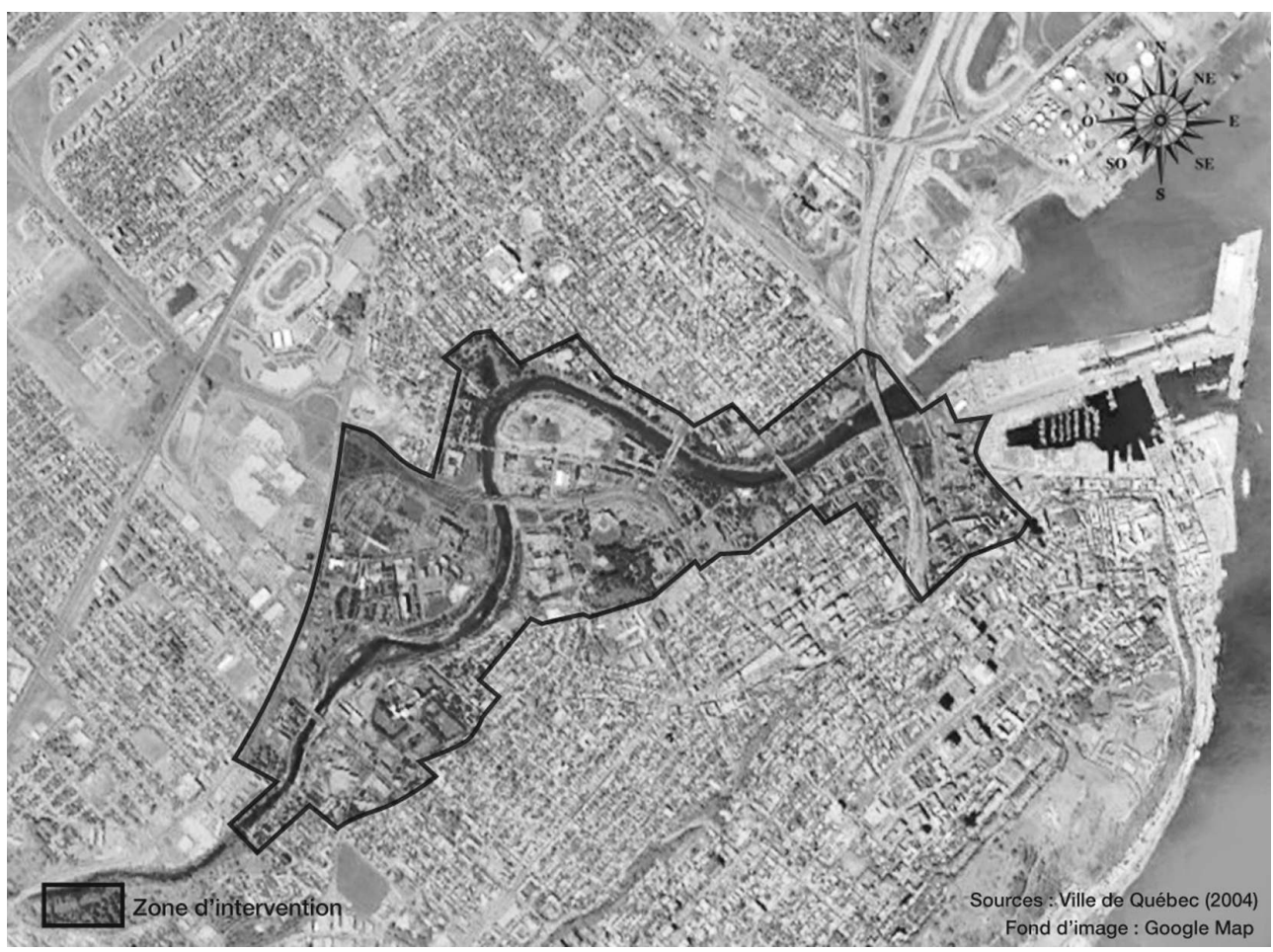

(Source : Ville de Québec, 2004)

18 Le projet de la Saint-Charles a par ailleurs tiré profit de l'expérience acquise quelques années plus tôt par la municipalité en matière de concertation lors du renouvellement urbain du quartier Saint-Roch. Symbole du déclin d'un centre-ville commerçant et industriel jusqu'au début des années 1990, Saint-Roch constitue aujourd'hui un exemple d'urbanisme négocié. Ainsi, suite à l'élection du Rassemblement populaire dirigé par JeanPaul L'Allier en 1989, la municipalité est parvenue à faire aboutir des projets en composant avec les acteurs locaux, après avoir tenté sans succès des années durant de moderniser le quartier à coups de "grands travaux " selon une approche descendante très controversée de la planification urbaine. La nouvelle équipe au pouvoir a mis l'accent sur la politique de consultation populaire, et insisté pour développer le quartier à partir du patrimoine déjà bâti, de son histoire, et non plus en y parachutant de grandes infrastructures ${ }^{7}$. Lors des consultations relatives au projet de "renaturation », la Ville a suivi ce précepte, quitte même à s'approprier des idées venues d'associations contestataires (comme le suggère le mémoire de l'association «Rivière Vivante », 1999, p.6). En agissant de la sorte, la municipalité a aussi bousculé l'organisation et les méthodes d'intervention de ses propres services techniques. 
Photo 1 : Le parc linéaire de la rivière Saint-Charles à Québec/Saint-Charles river park

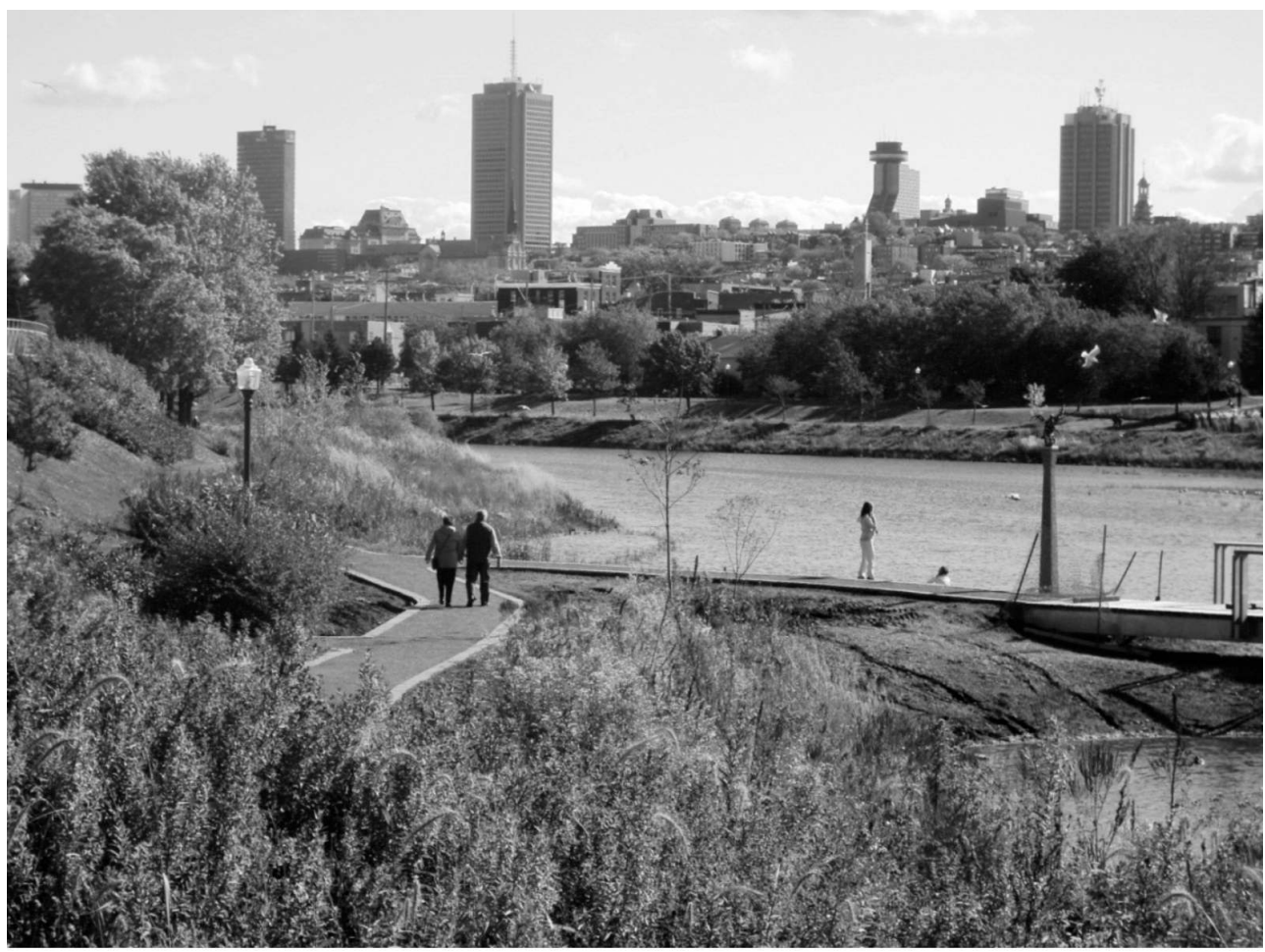

(cl. A. Brun, 2009)

19 Au total, la rupture voulue par la nouvelle municipalité de Québec a été rendue possible par un contexte politique favorable au cours des années 1990. Elle sous-tendait, comme l'a théorisé F. Scherrer (2004) au sujet de la valorisation des parcelles riveraines des rivières urbaines, qu'il était devenu légitime de limiter les pressions anthropiques sur un milieu naturel reconstitué et de considérer la qualité globale de la rivière comme facteur d'aménités urbaines.

\section{La « renaturation » de la basse Saint-Charles : une opération exemplaire?}

L'intérêt écologique de la " renaturation » de la section aval de la rivière Saint-Charles est manifeste compte tenu du retour de certaines espèces, même si le barrage Samson situé à Québec empêche la migration des poissons ${ }^{8}$. La qualité physico-chimique des eaux de surface tendra probablement à s'améliorer au cours des prochaines années grâce à l'amélioration progressive de la collecte des eaux pluviales, comme c'est le cas dans le reste du Québec (Duchesne, 2009). Par ailleurs, la création d'îlots, de lobes, de grèves et d'épis rocheux comptent aujourd'hui parmi les travaux les plus audacieux susceptibles d'être effectués dans un cours d'eau urbain forcément très "corseté ». Reste que cette opération, si elle marque un tournant en matière d'aménagement urbain au Québec, ne doit pas masquer le problème de l'étalement urbain qui caractérise les villes d'Amérique du Nord. L'urbanisation mal maîtrisée, en particulier en tête de bassin versant, peut avoir de graves conséquences sur la qualité de l'eau et, par ricochet, sur l'attractivité de la partie aval du bassin versant dans laquelle les pouvoirs publics ont beaucoup investi. 
Photo 2 : Le parc linéaire de la rivière Saint-Charles à Québec/Saint-Charles river park

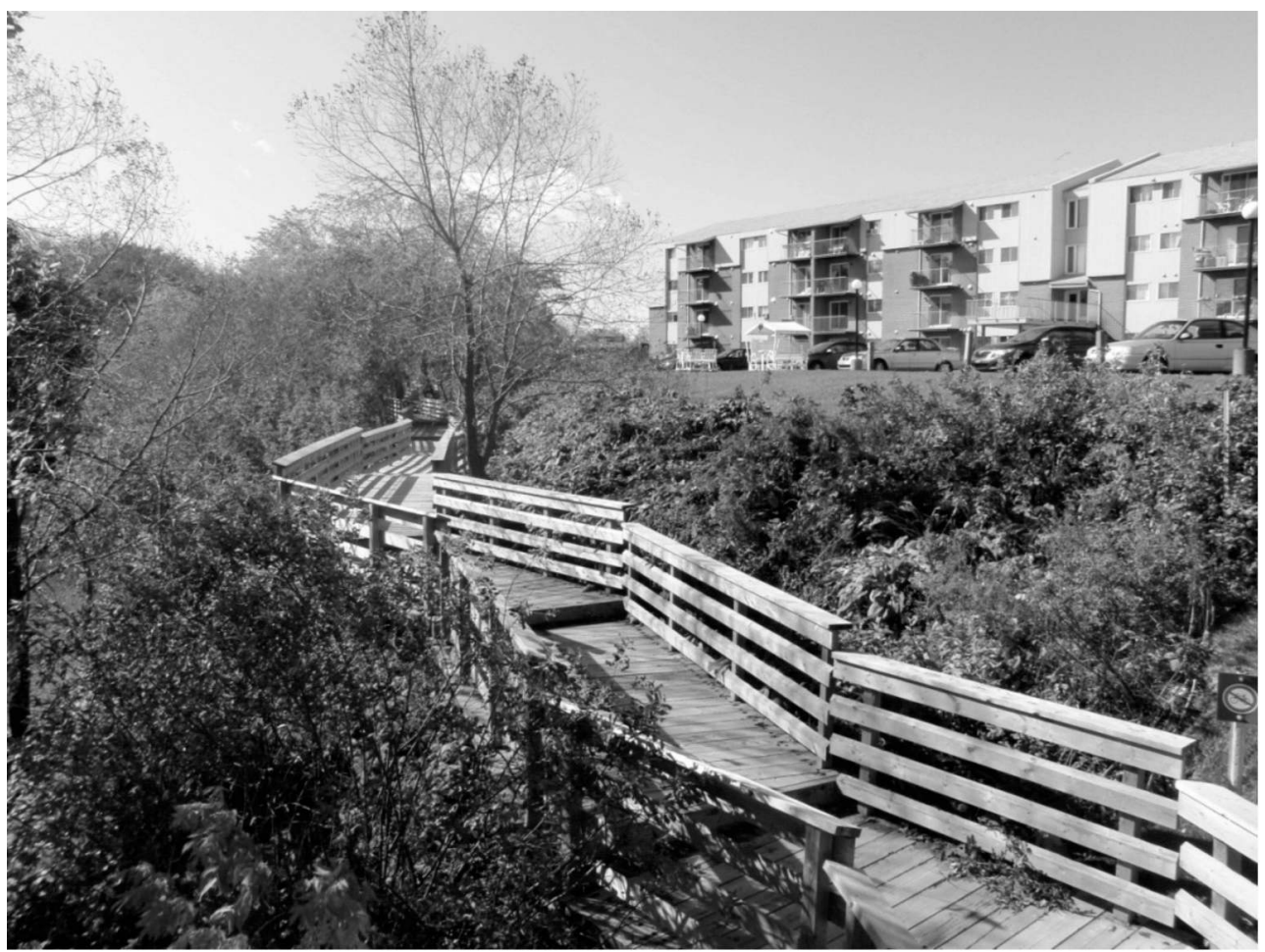

21 (cl. A. Brun, 2009)

\section{L'étalement urbain dans le bassin versant de la rivière Saint-Charles}

Dans la région de Québec, la superficie de la zone d'habitat urbain continu (mesurée selon les indications des cartes topographiques au $1 / 50000$ du ministère canadien des Ressources naturelles) a augmenté de $630 \%$ (de $36,9 \mathrm{~km}^{2}$ à $269,3 \mathrm{~km}^{2}$ ) entre 1950 et 2000, tandis que sa population ne crût que de $35 \%$ durant la même période (Mercier, 2006). Le développement périurbain connaît un essor dans certains secteurs localisés en tête de bassin. C'est le cas dans les sous-bassins de la rivière du Hibou ou du Ruisseau dans le bassin de la Saint-Charles (Portrait de bassin de la rivière Saint-Charles, 2007). Beaucoup de ménages aisés sont attirés par la proximité des pistes de ski et le cadre de vie des environs de Québec, assez bien reliés au centre-ville. «La logique d'aménagement des promoteurs lotisseurs, qui veut que le pavillon soit centré sur la parcelle, est [cependant] très consommatrice d'espace » reconnaît un élu local, qui ajoute aussitôt qu'il «faut répondre à la demande » (fig. 2).

Selon les associations de protection de l'environnement, les conséquences environnementales de l'étalement urbain dans le bassin versant de la Saint-Charles sont multiples : rejets domestiques à l'origine de la dégradation de la qualité des cours d'eau, érosion rivulaire accrue après défrichage du couvert boisé, destruction d'habitats fauniques... La plupart des lacs du bassin versant de la rivière Saint-Charles connaissent également des perturbations dues au développement résidentiel. Par exemple, à certaines périodes de l'année, la qualité bactériologique de l'eau du lac Durand ne permet pas la baignade. Les eaux du lac ne sont pas utilisées pour l'alimentation en eau potable, les 
résidences du secteur devant se munir de systèmes individuels d'approvisionnement. Les résidences ne sont pas toutes reliées à un système municipal d'évacuation des eaux usées et doivent donc avoir recours à l'assainissement autonome (Gérardin et Lachance, 1997; Bolduc, 2002). Or, il est impossible de vérifier la conformité de toutes les installations des particuliers.

En direction de l'aval, le bassin versant de la rivière Saint-Charles est de moins en moins forestier: aux perturbations causées par le développement résidentiel et récréotouristique s'ajoutent celles relatives à la multiplication des infrastructures routières. En 1973 par exemple, le redressement de la rivière du Berger sur $500 \mathrm{~m}$ au nord du boulevard Père-Lelièvre lors de la construction de l'autoroute du Vallon, puis la canalisation d'une autre partie de la rivière consécutive à la construction de l'autoroute de la Capitale, ont modifié l'écoulement naturel du cours d'eau, détruit l'habitat du poisson et accru l'endommagement des parcelles riveraines en cas de crue (Portrait de bassin de la rivière Saint-Charles, 2007). « Au cours des dix dernières années, 75 kilomètres de nouvelles rues ont été créées à Québec » précise l'association Vivre en Ville.

«Les projets de lotissements supplémentaires sont légions » explique un aménagiste en charge de la planification urbaine. La consultation publique qui devait être tenue en 2010 par la Commission consultative sur le schéma d'aménagement et de développement de la Ville de Québec portant sur le projet de règlement RAVQ 492 visant l'agrandissement du périmètre d'urbanisation et la modification de certaines aires d'affectation en témoigne 9 Le contrôle municipal consiste à régir ou à prohiber les usages du sol, les constructions, les ouvrages, les opérations cadastrales compte tenu de la proximité d'un cours d'eau ou d'un lac ou de tout autre facteur propre à la nature des lieux pouvant être pris en considération pour des raisons de sécurité publique ou de protection environnementale des rives, du littoral de tous les lacs et cours d'eau ou des plaines inondables.

La réglementation des plaines inondables peut aussi soumettre l'occupation du territoire à des contraintes sévères pour des raisons de sécurité publique. La réglementation d'urbanisme de la municipalité peut diviser le territoire de la municipalité, établir des catégories d'usages, de constructions ou d'ouvrages à prohiber ou à régir et décréter des prohibitions ou des règles qui varient selon les parties de territoire, les catégories ou toute combinaison de ces critères de sélection. Chacun toutefois s'accorde à dire, pour paraphraser un expert régional en aménagement, que « c'est la loi relative à la protection des terres agricoles qui constitue, aujourd'hui encore, le meilleur rempart face à l'urbanisation des espaces ruraux ».

L'absence de stratégie territoriale globale à l'échelle de l'aire métropolitaine est patente du point de vue des associations comme "Vivre en Ville», en particulier au sujet des déplacements interurbains. L'automobile reste le principal moyen de locomotion des milliers de banlieusards et les transports en commun se limitent au bus. De surcroit, la maison individuelle souvent située loin de l'hyper-centre demeure très recherchée par les ménages. Cela explique selon une élue de la banlieue de Québec que «faute de réellement maîtriser l'urbanisation du territoire, on a opté dans la région de Québec en faveur des constructions plus écologiques ». Il faut chercher dans la prédominance de la ville centre "qui veut faire la pluie et le beau temps", la difficulté de mise en œuvre d'une planification territoriale basée sur la solidarité entre les municipalités. De plus, « l'espace ne manque pas, c'est pourquoi il est peu commode de changer les pratiques des promoteurs et les logiques municipales [...] : la densification fait peur en Amérique du Nord! » (« Vivre en Ville »). 
Dans ce contexte, les principes de la gestion de l'eau par bassin versant peinent à s'imposer aux acteurs de l'aménagement malgré la mise en place d'une gouvernance locale de l'eau théoriquement « intégratrice ». En simplifiant beaucoup, le principal écueil est le suivant: la gouvernance locale de l'eau ne constitue en rien un levier pour l'aménagement aux yeux des élus locaux.

Figure 3 : L'urbanisation dans le bassin versant de la rivière Saint-Charles /Urbanization in watershed
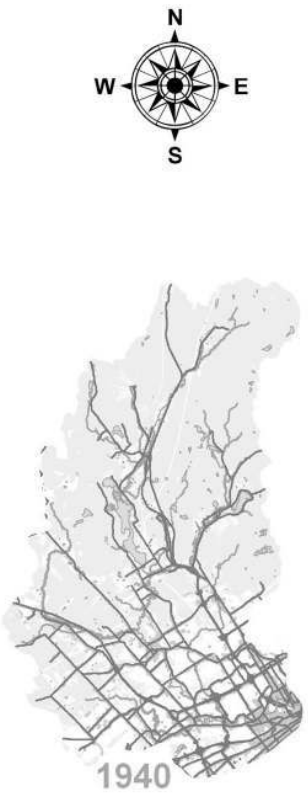

$0 \quad 5$

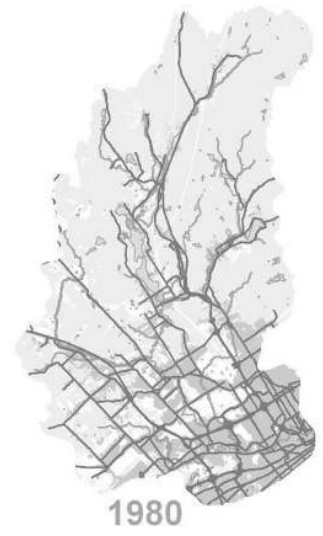

1980
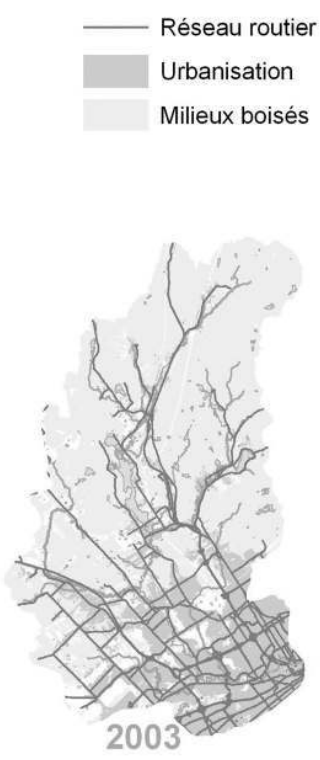

20 Kilomètres

(source : Plan directeur de l'eau, Conseil du bassin-versant de la rivière Saint-Charles, 2009)

\section{Le rôle limité du conseil de bassin de la rivière Saint-Charles}

Du milieu des années 1960 jusqu'à la fin des années 1990, la gestion des ressources en eau et le statut de l'eau ont fait l'objet de nombreux débats sans toutefois que ceux-ci ne débouchent sur des avancées concrètes. La Politique Nationale de l'Eau du Québec (PNE) présentée en 2002 par le gouvernement de Bernard Landry (Parti Québécois) à l'issue d'une série de consultations organisées entre 1997 et 2000 marque par conséquent un tournant. La PNE - influencée à la fois par les modes d'action publique anglo-saxons et français -, s'est en particulier traduite par l'installation d'organisations de bassin versant dans 33 bassins versants alors jugés prioritaires par les pouvoirs publics (Brun, 2009). Leur rôle est central car elles concourent à la planification dans le domaine de l'eau (Plans Directeurs de l'Eau) et à l'expérimentation de nouveaux instruments contractuels (Contrats de bassins).

Un Plan Directeur de l'Eau (PDE) comprend un diagnostic de bassin versant et les interventions envisagées. Le contrat de bassin est le volet opérationnel du PDE. Le ministère de l'Environnement du Québec l'a présenté en 2002 comme une convention dans laquelle sont consignées les actions des différentes parties qui désirent s'impliquer - 
selon leurs propres volontés, droits ou responsabilités - dans la protection, la restauration ou la mise en valeur du bassin. Il comprend les détails des actions à entreprendre, les coûts, les noms des maîtres d'œuvre et l'échéancier. Depuis 2004, certains de ces contrats de bassin peuvent prendre la forme de simples protocoles d'entente. Mais lorsque les actions des PDE s'y prêtent, ils peuvent également être des contrats en bonne et due forme, conclus entre deux ou plusieurs parties, et donc avoir une valeur juridique (Choquette, 2008). Les organisations de bassin sont par conséquent sensées jouer un rôle déterminant au niveau de la planification territoriale.

L'État ne s'est pas pour autant désengagé, au contraire. En 2007, le gouvernement du Québec a renforcé les actions portant sur la lutte contre les cyanobactéries (MDDEP, 2007) et créé des «Zones de gestion intégrée » (2009) de façon à couvrir l'ensemble du Québec méridional tout en reconnaissant le rôle croissant des organismes de bassin. Le législateur a de surcroît adopté le 11 juin 2009 la Loi affirmant le caractère collectif des ressources en eau et visant à renforcer leur protection. Cette loi comprend en particulier un article sans pareil au Canada qui affirme que les eaux sont des « ressources qui font partie du patrimoine commun de la nation québécoise ». Elle répond aussi - non sans ambiguïtés - à certaines propositions des rapports des Commissions Legendre en 1975 et Beauchamp en 2000 et met en œuvre des engagements de la PNE (Halley et Gagnon, 2009). La politique de l'eau au Québec s'est donc mise en place malgré l'alternance politique survenue en 2004 au sein de l'exécutif québécois.

32 Le gouvernement du Québec, principal financeur des organismes de bassin versant, a cependant sous-estimé les besoins des organisations de bassins parfois inopérantes faute d'argent (en dépit de l'augmentation récente des subventions gouvernementales). Il en résulte des difficultés tant en matière de fonctionnement (nombre de déplacements limités pour les salariés, accès insuffisants aux colloques et aux rendez-vous socioprofessionnels, etc.) qu'en matière de recrutement (seuls les employés peu expérimentés peuvent être embauchés compte tenu des salaires proposés ${ }^{10}$. De plus, la fragilité financière des organismes de bassin versant a tendance à accroître la concurrence entre eux (prestation de services relatifs à la nature, appels à projets pour l'obtention de subventions de tel ou tel fond), de sorte que la constitution même d'un groupe d'intérêt uni autour de la gestion intégrée de l'eau par bassin versant paraît illusoire (Brun, 2009). Le Gouvernement du Québec s'est, pour beaucoup, déchargé de la gestion de l'eau au prétexte de favoriser la gouvernance locale de la ressource ${ }^{11}$.

Leur statut d'organisme à but non lucratif (OBNL) est de surcroît inapproprié : « Un OBNL a normalement son autonomie, des membres qui définissent sa mission, ses mandats et ses structures et qui élisent leurs représentants au conseil d'administration » (Raîche, 2008). La dépendance des organismes de bassin versant vis-à-vis du Gouvernement les discrédite enfin aux yeux d'autres associations de protection de la nature. Ces dernières sont souvent plus anciennement implantées et se disent à la fois plus autonomes financièrement et plus légitimes politiquement car elles n'ont pas attendu une décision gouvernementale pour se constituer et agir. Ainsi, de l'association gestionnaire du Parc de la Rivière-des-Milles-Îles aux pêcheurs sportifs de la Gatineau, on s'interroge sur le rôle et surtout sur les moyens des organismes de bassin versant jugés en inadéquation avec leurs objectifs. Était-il nécessaire, en d'autres termes, de créer des structures associatives supplémentaires à celles qui existaient déjà, et ce d'autant que certaines possèdent de l'expérience dans le domaine de la gestion des rivières quand d'autres sont allées jusqu'à amorcer une gestion par bassin ${ }^{12}$ ? 

l'environnement (APEL) a été créée dès 1980 par des bénévoles riverains du lac SaintCharles, 20 ans avant la création du Conseil de bassin de la Saint-Charles. Depuis 1997, l'APEL s'est peu à peu "professionnalisée » puisqu'elle compte désormais plusieurs salariés. Son budget, qui oscille entre $250000 \$$ et $500000 \$$ par an, repose sur des subventions des municipalités, de la fondation de la faune et du ministère du Développement Durable, de l'Environnement et des Parcs. Suite à la mise en place de la politique nationale de l'eau du Québec, il a été question que l'APEL modifie ses statuts de façon à devenir un organisme de bassin versant. Toutefois ses administrateurs s'y sont opposés, faisant valoir que la composition du conseil d'administration et le mandat de ces organismes peut déboucher sur des conflits d'intérêts internes et un risque d'éparpillement qui nuirait à son action. La stratégie de l'APEL a donc consisté à collaborer avec le Conseil de bassin de la rivière Saint-Charles tout en garantissant son indépendance et ses propres objectifs. Outre les habituelles actions de sensibilisation, l'APEL réalise des travaux et assure la gestion des «marais du Nord » du lac Saint-Charles dont elle est pour partie propriétaire à part égale avec la Ville de Québec. Plusieurs élus locaux et des représentants du service de l'urbanisme de la Ville de Québec ont souligné le rôle de " sentinelle » exercé par l'APEL : « les développeurs savent maintenant ce qu'ils peuvent faire ou pas, car les associations environnementales sont vigilantes» (un représentant du service de l'environnement de la Ville de Québec). d'observateur que d'acteur depuis sa création en 2003, parce que le Gouvernement le cantonne à ce rôle. D'après ses statuts, le Conseil de bassin de la rivière Saint-Charles a pour principale mission d'assurer la concertation des usagers et des gestionnaires, à l'échelle du bassin versant de la rivière Saint-Charles. Il fait la promotion de la protection et de la mise en valeur du territoire dans une perspective de développement durable par le biais d'une approche intégrée de gestion des ressources. Il favorise également la coordination des activités exercées par les utilisateurs des ressources dans une approche de prévention et de résolution des conflits potentiels d'usages. Enfin, il fait valoir, par le biais de recommandations auprès des structures locales, régionales et nationales, les intérêts et les préoccupations des intervenants du bassin versant. Le Conseil de bassin de la rivière Saint-Charles est surtout responsable de l'élaboration du PDE et doit veiller à sa mise en œuvre, à son suivi et à son évaluation.

Le Conseil mène toutefois des actions qui n'ont pas la portée de celles de l'APEL auprès des acteurs locaux. Tout juste est-il en mesure comme n'importe quelle autre association de pointer du doigt les éventuelles incohérences des politiques municipales - dans une certaine limite néanmoins puisque une partie de ses membres, élus ou aménagistes, représentent des municipalités. Au reste, la Ville de Québec ne veut pas déléguer de compétences à l'organisme de bassin versant car en interne les syndicats des agents municipaux s'y opposent fermement: la gestion de l'eau est du ressort du pouvoir municipal. Ce faisant le Conseil ne peut exercer ni le rôle de maitre d'ouvrage de travaux, ni celui de maître d'ouvrage délégué, s'il en avait les moyens humains, matériels et financiers. Le Conseil n'intervient pratiquement pas non plus lors de l'élaboration des outils de planification urbaine.

$\mathrm{Au}$ total, les associations environnementales sont parfois associées les unes aux autres et travaillent en réseaux lorsqu'elles ne sont pas mises en concurrence par leurs financeurs. Leurs collaborations ponctuelles sont renforcées par leur proximité géographique 
(plusieurs d'entre elles sont situées dans une maison des associations), la formation initiale commune de leurs salariés et la présence de personnalités locales impliquées dans leurs conseils d'administration respectifs. Elles dressent surtout un constat partagé : même si l'eau est abondante dans le bassin versant de la rivière Saint-Charles, l'étalement urbain risque de masquer d'ici peu les efforts consentis par les municipalités pour sauvegarder la ressource en eau et favoriser le retour des usagers au bord des cours d'eau urbains, comme c'est le cas avec la "renaturation » de la basse Saint-Charles. Chacune conserve cependant ses propres objectifs et stratégies. Aucune d'entre elles ne considère avoir été l'artisan de la « renaturation ${ }^{13}$ ».

L'incapacité des structures associatives à être maître d'ouvrage dans l'aménagement et la gestion des cours d'eau et leur manque de moyens financiers les relègue au second plan aux yeux des élus locaux et des développeurs : certains ne connaissent même pas leur existence. Autrement dit, la gouvernance locale de l'eau telle qu'elle s'est peu à peu organisée dans le bassin versant depuis les années 2000 demeure peu influente par rapport aux exécutifs municipaux plus anciens et politiquement plus légitimes : seule une intercommunalité de projet articulant eau et territoire pourrait pallier cet écueil, le préalable étant d'accroître la porosité entre d'une part la planification dans le domaine de l'eau et d'autre part la planification territoriale.

\section{Les schémas d'aménagement, des outils complémentaires aux PDE ?}

La Loi sur l'aménagement et l'urbanisme (LAU), adoptée en 1979, n'a pas de libellé centré spécifiquement sur la gestion de l'eau par bassin versant. Elle encadre en revanche l'élaboration du schéma d'aménagement et de développement réalisé par chaque Municipalité Régionale de Comté (MRC), lesquelles peuvent faciliter l'articulation entre planification urbaine et planification dans le domaine de la gestion de l'eau. L'article 5 de la LAU fixe le contenu obligatoire du schéma d'aménagement et de développement alors que l'article 6 présente un contenu facultatif. Un schéma se compose généralement d'un état de la situation, d'un exposé des choix d'aménagement (stratégie de développement, concept d'aménagement privilégié et affectation des sols) et d'un recueil de règles et critères relatifs à la destination des sols en particulier. Chaque MRC étant libre d'élaborer son schéma selon la structure de son choix, elle est à tout le moins en mesure de prendre en compte l'eau dans le cadre de cet exercice prospectif. De plus, certaines dispositions de la Loi sur les compétences municipales (2006) offrent de nouvelles compétences aux MRC et aux municipalités en matière de protection de l'environnement.

Le schéma d'aménagement et de développement n'est pas cependant un outil idéal selon les aménagistes pour amorcer un processus d'intégration des enjeux de l'eau dans l'action municipale. Sa dimension réglementaire limiterait les possibilités d'expérimentation et son champ géographique d'application se superpose rarement avec celui du PDE. De plus, les calendriers diffèrent. D'une part, environ 7 des 33 organismes de bassins versants prioritaires ont leur PDE approuvé mais avec le redécoupage du Québec méridional en zones, des ajustements sont prévisibles. D'autre part, environ cinq MRC élaborent leur schéma d'aménagement et de développement de troisième génération alors que près de la moitié des autres MRC n'ont pas encore finalisé un schéma d'aménagement et de développement de seconde génération. Cette situation complexifie l'arrimage des contenus, et ce d'autant que les aménagistes ne sont pas systématiquement impliqués 
dans les conseils d'administration des organismes de bassin... Enfin, pour entrer en vigueur, un schéma doit recevoir un avis gouvernemental (40 ministères et organismes sont au total concernés) attestant sa conformité aux orientations du Gouvernement du Québec en matière d'aménagement du territoire. Or, l'évaluation du schéma relève du ministère chargé des affaires municipales, mais celle du PDE relève du ministère chargé du Développement durable, de l'Environnement et des Parcs.

41 Pour pallier l'absence d'articulation entre schéma d'aménagement et de développement et plan directeur de l'eau, la Corporation de l'aménagement de la rivière l'Assomption (CARA, située à Joliette à proximité de Montréal) a initié, avec les 11 MRC de son territoire, et en partenariat avec les ministères chargés de l'Environnement et des Affaires municipales une démarche originale. Celle-ci s'est traduite par l'expérimentation de cadres d'actions communs aux aménagistes et aux animateurs de la CARA à différents échelons : un cadre régional de gestion des bassins versants qui sera intégré au schéma de la MRC, un cadre municipal de gestion de l'eau qui sera intégré au plan d'urbanisme de la municipalité, des plans d'actions de lacs élaborés par les municipalités avec l'aide des organisations de bassin et les association de riverains (sans l'accord desquelles les actions seraient très limitées fautes d'accès aux lacs) et des plans d'actions des cours d'eau élaborés par les MRC concernées en collaboration avec les organismes de bassin (Fafard, 2009).

Néanmoins, nombre de municipalités québécoises ne jouent pas le jeu en libérant des parcelles boisées ou agricoles à la construction sous la pression des promoteurs immobiliers d'après "Canards Illimités", fondation particulièrement attentive à l'évolution de l'usage des zones humides dans le Québec méridional ${ }^{14}$. La pression exercée sur les zones riveraines des plaines d'inondation s'intensifie en effet. Environ 4000 hectares de milieux humides ont été perdus au cours des 50 dernières années le long des rives du Saint-Laurent, entre Cornwall et Matane selon le Gouvernement du Québec (2002).

43 L'autre difficulté est due au fait que «l'aménagement du territoire remonte seulement aux années 1980 : c'est un concept récent que les municipalités ont eu de la peine à s'approprier à cause d'un certain laxisme au niveau local » comme le souligne Fabienne Mathieu, interviewée alors qu'elle était à la fois présidente du Conseil de bassin de la rivière Saint-Charles et directrice de la Municipalité des cantons unis de Stoneham-etTewkesbury. Il faut enfin que les conditions de la coopération politique soient réunies, ce qui n'est pas le cas dans le bassin de la rivière Saint-Charles où il n'y a pas de relations constructives entre les maires de Stoneham et de Québec, ajoute Fabienne Mathieu ${ }^{15}$. Dans la région de Québec « l'élaboration du schéma d'aménagement de la Communauté métropolitaine a pris cinq ans de retard ${ }^{16}$ ", ce qui témoigne de l'extrême difficulté de travailler à l'échelle métropolitaine explique Alain Marsolais, du ministère chargé des affaires municipales.

Les règlements d'urbanisme ${ }^{17}$ sont faits de telle sorte que l'immense majorité des projets immobiliers même les plus controversés aux plans environnemental et architectural sont conformes : «on ne peut donc pas blâmer les inspecteurs » chargés de l'instruction des permis de construire, d'autant qu'ils sont en « sous effectifs dans plusieurs municipalités du bassin» (APEL). La consommation d'espace, et, en corollaire la perturbation des écosystèmes aquatiques, sont donc paradoxalement limitées grâce à la Loi sur la protection des terres agricoles. De surcroit, ce sont les pénuries périodiques d'eau potable et les difficultés rencontrées en matière d'assainissement qui ont protégé de 
l'urbanisation l'île d'Orléans convoitée par les promoteurs immobiliers et les particuliers explique Fabienne Mathieu. Aussi peut-on en conclure que les outils de planification en général, et ceux dans le domaine de l'eau en particulier, jouent pour l'heure un rôle limité dans l'évolution du rapport ville-campagne, et, par conséquent, dans le traitement préventif des problèmes de l'eau.

L'adoption le 8 novembre 2010 d'un Règlement de contrôle intérimaire visant à limiter les interventions humaines dans les bassins versants des prises d'eau de la Ville de Québec installées dans la rivière Saint-Charles et la rivière Montmorency montre toutefois combien le thème de la préservation de la ressource en eau préoccupe désormais les pouvoirs publics à l'échelle métropolitaine. Ce règlement n'a certes pas pour but d'interdire le développement pavillonnaire mais il impose des prescriptions techniques et architecturales précises aux propriétaires en matière d'assainissement afin que soient mieux protégées les prises d'eau de la région de Québec ${ }^{18}$. Marque-t-il un infléchissement de la politique municipale en faveur d'une gestion plus intégrée de l'eau ? Peut-il aboutir à une réelle articulation entre les outils de planification relatifs à l'eau et à l'urbanisme ?

\section{Conclusion}

La Politique nationale de l'eau du Québec adoptée en 2002 a posé le principe de la gestion intégrée de l'eau par bassin versant et favorisé la création d'organismes chargés de planifier la protection de la ressource. Les municipalités restent toutefois les principaux artisans de la politique locale de l'eau compte tenu des attributions et des moyens qui sont leurs sont dévolus. En somme, la recomposition du paysage institutionnel qui s'est opérée dans le domaine de l'eau au Québec n'a pas fondamentalement modifié la prépondérance de la politique municipale sur la gouvernance de l'eau.

47 L'exécutif local - incarné par le maire - a toujours joué un rôle central en matière d'aménagement urbain comme l'illustre le cas de la basse rivière Saint-Charles, d'abord « bétonnée » dans les années 1960 par la volonté d'un maire, puis « renaturée » trente ans plus tard par la volonté d'un autre, et ce, dans le même objectif, à savoir rendre le cours d'eau à ses habitants. Le Conseil de Bassin de la rivière Saint-Charles à peine créé a suivi en « spectateur » la fin du chantier de « renaturation » engagé au milieu des années 1990.

La perspective de mettre en scène la basse Saint-Charles - au même titre que les bords du Saint-Laurent - lors des festivités du $400^{\mathrm{e}}$ anniversaire de Québec a légitimé l'entreprise de la municipalité aux yeux d'une partie des habitants, facilité peut-être le déblocage de crédits publics des différents paliers de gouvernement, atténué les conflits entre les services de la ville et permis le respect du calendrier initial.

La Ville a su tirer profit du processus de concertation qu'elle a initié au point d'être vivement critiquée par plusieurs associations qui se sentaient dépossédées de «leurs » projets au profit de la municipalité. La concertation a, de toute évidence, stimulé le débat public et elle s'est soldée par une nette évolution du projet initial : le génie écologique s'est imposé de manière progressive aux bureaux d'études techniques, au service de l'urbanisme de la Ville de Québec, aux entreprises de travaux publics intéressées et aux élus locaux.

50 La « renaturation » des rivières urbaines est un thème largement traité aujourd'hui en sciences sociales et urbaines (Mahaut, 2009; Ultsch, 2010) dans la mesure où la «renaturation» inscrit physiquement dans la ville le changement de paradigme qui 
s'opère depuis les années 1970 en termes de relation société-nature. Les maires ont désormais des velléités en ce domaine; les retours d'expériences ne manquent plus, en particulier aux États-Unis, en France, en Allemagne, au Luxembourg et en Suisse. La "renaturation" masque cependant les effets néfastes sur la ressource en eau d'un étalement urbain non maîtrisé. Faire réapparaître la nature en ville n'exonère pas les municipalités de mettre en œuvre une planification territoriale et urbaine plus rationnelle doublée si nécessaire de dispositifs incitatifs (telle qu'une taxe sur les surfaces imperméabilisées) destinés à limiter l'urbanisation des secteurs stratégiques. Dans cette optique, le plan directeur de l'eau du bassin de la Saint-Charles (désormais achevé) et l'expertise de terrain des acteurs de l'eau se révéleront sans doute utiles à la Ville de Québec dans sa stratégie de sauvegarde des réserves d'eau potable et de conservation de la qualité du cadre de vie.

51 Je remercie les personnes ayant participé à cette recherche à commencer par Jean-Paul Haghe (Université de Rouen), Gabriel Rioux (université du Québec à Montréal) et Christine Simoens (Sogreah). Merci également à Pierre Dulude, pour son expertise écologique, au Conseil du bassin de la Saint-Charles pour sa collaboration et à Armelle Brun pour l'analyse du draft.

\section{BIBLIOGRAPHIE}

BEAULIEU M., 2006. La rivière Saint-Charles : dégradation et renaissance d'une rivière urbaine, FrancVert, Vol.3/1, en ligne, [http://www.francvert.org/pages/31dossierlarivieresaintcharlesdegrad.asp ], $13 \mathrm{p}$.

Bolduc F., 2002. Diagnose des lacs Durand et Trois-Lacs, Cantons-Unis de Stoneham et Tewkesbury, rapport de Pro Faune, 56 p. (3 annexes).

BOUTET G., 2006. Le changement de forme des berges de la rivière Saint-Charles à Québec : l'explication de l'approche culturelle de la géographie, mémoire de maîtrise, Université Laval, Québec, Canada, 162 p. BRUN A., 2009. L'approche par bassin versant : le cas du Québec, Options Politiques, juillet-août, p. $36-42$.

BRUN A., 2010. Les contrats de rivière en France : enjeux, acteurs et territoires, Cahiers de droit de l'Université Laval, vol. 51, nº 3-4, p. 679-704.

BRUn A., LASSERRE F. (dir.), 2006. Politiques de l'eau. Grands principes et réalités locales, Québec, Canada, Éditions PUQ, 408 p.

BUNUSEVAC M., 2007. Renaturation des cours d'eau, restauration des habitats humides, Gouvernement du Grand Duché du Luxembourg, 97 p.

CHAMBRE DE COMMERCE DE QUÉBEC, 1962. Mémoire sur la rivière Saint-Charles, Québec, La Chambre, $33 \mathrm{p}$. 
Choquette C., 2008. Le contrat de bassin : un outil de gestion à géomètrie variable, dans $\mathrm{C}$ HOQUETTE C., LETOURNEAU A. (dir.), Vers une gouvernance de l'eau au Québec, Québec, Canada, Éditions MultiMondes, p. 281-298.

CONSEIL DU BASSINT-VERSANT DE LA RIVIÈRE SAINT-CHARLES, Portrait de bassin de la rivière Saint-Charles, $2007,216 \mathrm{p}$.

DUCHESNE S., 2009. La gestion des eaux usées et pluviales en milieu urbain au Québec : problématiques et défis, La gouvernance de l'eau dans les Amériques, Observatoire des recherches internationales sur l'eau, Université Laval, Québec, Canada, 15-16 octobre.

DuMONT J.-P., 1998. Une rivière dans la ville ; l'usage urbain de la rivière Saint-Charles : origines et perspectives, mémoire de maîtrise, Université Laval, Québec, Canada, 88 p.

FAFARD D., 2009. Outils complémentaires à la gestion par bassin : les schémas d'aménagement, rapport de l'Association des biologistes du Québec, n. p.

GERARDIN V., LACHANCE Y., 1997. Vers une gestion intégrée des bassins versants. Atlas du cadre écologique de référence du bassin versant de la rivière Saint-Charles, Québec, Canada. Ministère de l'Environnement et de la Faune/Ministère de l'Environnement Canada, 58 p.

GHIOTTI S., 2007. Les territoires de l'eau. Gestion et développement en France, Paris, CNRS, coll.

«Espaces et milieux », $248 \mathrm{p}$.

HALLEY P., GAGNON C., 2009. Le droit nouveau de l'eau au Québec, CRDE/Université Laval-Gaïapresse, 3 p. [http://www.gaiapresse.ca/fr/analyses/index.php?id=109] consulté le $1^{\mathrm{er}}$ juin 2010.

LEBEL J.-M., RoY A., 2000. Québec 1900-2000. Le siècle d'une capitale, Québec, Canada, Éditions MultiMondes et Commission de la capitale nationale du Québec, 176 p.

LEMONIER M., 2003. Les berges, territoires de projets, Diagonales, n 163, p. 39-42.

MAHAUT V., 2009. L'eau et la ville, le temps de la réconciliation. Jardins d'orage et nouvelles rivières urbaines, thèse de doctorat en sciences Appliquées, département d'architecture, d'urbanisme et de génie civil environnemental, Université Catholique de Louvain, Belgique, 430 p.

MALAVOI J.-R., 2010. La renaturation des cours d'eau : enjeux et études de cas, Séminaire sur les politiques de l'eau, master d'action publique, PontsParisTech, 6-7 avril 2010.

MALAVOI J.-R., BRAVARD J.-P., 2010. Élements d'hydromorphologie fluviale, Onema, 224 p.

MERCIER G., 2006. La norme pavillonnaire : mythologie contemporaine, idéal urbain, pacte social, ordre industriel, moralité capitaliste et idéalisme démocratique, Cahiers de géographie du Québec, vol. 50, n 140, p. 207-239.

Ministère du DÉveloppement duRABle, de L'EnVIRONNEMENT et Des PARCS, 2007. Plan d'intervention détaillé sur les algues bleu-vert, Gouvernement du Québec, 10 p.

Ministère du DÉVeloppement durable, de l'EnVironnement et Des Parcs, 2010. Règlement sur la redevance exigible pour l'utilisation de l'eau, Gouvernement du Québec, [http://www.mddep.gouv.qc.ca/ eau/redevance/reglement.htm].

PAULIN M., 2001. Mise en valeur et renaturalisation de la Saint-Charles, TSM, n 3, p. 29-37.

Pouliot Y., 2005. Le corridor naturel de la Saint-Charles, un milieu exceptionnel, Le naturaliste canadien, vol. $129, \mathrm{n}^{\circ} 2$, p. $78-81$.

PRONOVOST R., 2009. La renaturalisation des berges de la rivière Saint-Charles, Rendez-vous sur la gestion intégrée des ressources en eau, Université de Sherbrooke, Québec, 1-3 juin. 
RAîcHE J.-P., 2008. Gestion intégrée de l'eau au Québec : bilan et prospective. Approches concrètes pour une nouvelle gouvernance, dans CHOQUETTE C., LETOURNEAU A. (dir.), Vers une gouvernance de l'eau au Québec, Québec, Canada, Éditions MultiMondes, p. 261-280.

RIVIÈRE VIVANTE, 1996. Pré-concept d'aménagement proposé pour la rivière Saint-Charles entre les ponts Scott et Samson, non paginé.

RIVIÈRE VIVANTE, 1999. Le cas de la rivière Saint-Charles à Québec : le rôle d'un organisme de rivière, succès et embuches, Mémoire déposé par l'association au bureau d'audiences publiques sur l'environnement à l'occasion de la Consultation publique sur la gestion de l'eau au Québec, 46 p. SERVICE DE L'URBANISME DE LA VILLE DE QUÉBEC, 1973. Sauvegarde des abords des rivières Saint-Charles et Duberger, Division de l'Aménagement, Ville de Québec, 86 p.

SCHERRER F., 2004. L'eau urbaine ou le pouvoir de renaturer, Cybergéo : European Journal of Geography, Séminaire de recherche du GDR Rés-Eau-Ville (CNRS 2524) «L'eau à la rencontre des territoires », Montpellier, France, 27-29 mai 2004 [http://cybergeo.revues.org/1496].

UlTSCH J., 2010. Les temporalités de l'interface ville-rivière à travers le cas de Saint-Étienne et du Furan, Géocarrefour, vol. 85, n 3, p. 209-219.

VILLE DE QUÉBEC : RAPPORT DES COMMISSAIRES, 1996. Commission pour la mise en valeur du projet de dépollution et de renaturalisation de la riviere Saint-Charles, Ville de Québec, 83 p.

\section{ANNEXES}

Association pour la protection de l'environnement du lac Saint-Charles et des Marais du Nord (APEL)

Canards Illimités Canada (CIC)

Communauté métropolitaine de Québec (CMQ)

Corporation de l'aménagement de la rivière l'Assomption (CARA)

Loi sur l'aménagement et l'urbanisme (LAU)

Loi sur la protection du territoire agricole (LPTA)

Loi sur la qualité de l'environnement (LQE)

Lois refondues du Québec (LRQ)

Ministère de l'Agriculture, des Pêcheries et de l'Alimentation du Québec (MAPAQ)

Ministère des Affaires municipales, des Régions et de l'Occupation du territoire (MAMROT)

Ministère du Développement durable, de l'Environnement et des Parcs (MDDEP)

Municipalité régionale de comté (MRC)

Organisme à but non lucratif (OBNL)

Organisme de bassin (OBV)

Plan directeur de l'eau (PDE)

Politique nationale de l'eau du Québec (PNE)

Région métropolitaine de recensement (RMR) 


\section{NOTES}

1. 1. Souvent recalibrés et exploités, et ce généralement depuis plusieurs siècles (moulins, creusement du chenal de navigation, endiguement pour lutter contre les crues, etc.), les cours d'eau connaissent aujourd'hui d'importants dysfonctionnements. «La banalisation de leurs berges et de leurs lits perturbe leurs capacités de régulation des pollutions, d'alimentation des nappes et de régulation des crues, fonctions que les milieux humides assument gratuitement quand ils sont en équilibre naturel » résume le bureau d'études Bunusevac (2007). Comme le souligne J. R. Malavoi (2010), le terme de "renaturation" recouvre une grande variété d'interventions, allant du «reméandrage » d'une rivière autrefois canalisées jusqu'à des travaux infiniment plus modestes (déminéralisation partielle de lits artificiels de petits ruisseaux urbains). L'hydromorphologie fluviale appliquée est récente (Malavoi et Bravard, 2010), ce qui explique le tâtonnement des maîtres d'ouvrages et l'instabilité de la définition du terme de "renaturation» (les québécois utilisent préférentiellement le terme de "renaturalisation »). Selon nous, la mutabilité des parcelles riveraines du cours d'eau, la présence d'une maîtrise d'ouvrage unique et un financement assuré sur une décennie ou plus (études, travaux, évaluation) constituent des préalables indispensables à la conduite d'opérations d'aménagement de cette nature.

2. «IDEAUX " est l'un des douze projets interdisciplinaires retenus au titre du programme «Eaux \& Territoires » (2008-2011). L'équipe est, au reste, subventionnée au titre du Programme d'échanges franco-québécois Samuel de Champlain (2009 et 2010), soutenu par le ministère des relations internationales du Québec et le Consulat Général de France à Québec. Elle bénéficie aussi d'une participation du centre Jacques Cartier en 2009.

3. Cette séquence a été précédée, d'une part, de l'analyse de documents de planification urbaine et territoriale et de l'examen des outils de planification dans le domaine de la gestion de l'eau, et, d'autre part, d'une étude minutieuse des dynamiques d'aménagement dans les secteurs étudiés depuis une cinquantaine d'années. Cette démarche a été entreprise en collaboration avec des organismes locaux tels que le Comité du bassin versant de la rivière Gatineau au sujet du ruisseau de la Brasserie à Gatineau ou encore le Conseil de bassin de la rivière Saint-Charles dans le secteur d'étude de Québec.

4. Les urbanistes Gréber, Fiset et Bédard prévoyaient la mise en place d'un réseau d'autoroutes pour répondre au développement périurbain et au réaménagement des raccords avec les grandes routes provinciales. "Les urbanistes suggèr[aient] trois [...] routes à six voies [chacune], l'une reliant Québec à sa banlieue nord (ce qui deviendra l'autoroute laurentienne), un autre servant de voie d'accès le long du fleuve (le futur boulevard Champlain), la troisième longeant le côté sud de la rivière Saint-Charles et la voie ferrée du quartier Saint-Sauveur jusqu'à la gare du Palais (et qui sera remplacée par le prolongement du boulevard Charest) » (Lebel et Roy, 2000, p. 66).

5. Les propos sont rapportés par Lucien Bouchard pour Le Soleil dans l'édition du 21 novembre 1966: «Lamontagne: Québec deviendra l'une des plus belles capitales du monde » cité par J.-P. Dumont (1998).

6. L'accès au fleuve le long du Boulevard Champlain a également mobilisé les pouvoirs publics. Le Conseil des ministres a agi très vite afin d'éviter la spéculation. Dès le début 
juin 2000, il a adopté un décret qui a imposé une réserve de terrains "pour fins publiques ». Il a ainsi bloqué toute spéculation sur 360000 mètres carrés de terrains le long du boulevard Champlain en bordure du fleuve jusqu'au pont de Québec. Ce décret accordait un délai de quatre ans pour permettre au gouvernement de racheter les terrains nécessaires et de planifier une future " promenade du littoral » qui deviendra en 2008 la Promenade Champlain (source: [http://quebec.blog.lemonde.fr/2008/12/] consulté le 2 février 2011).

7. "Jean-Paul L'Allier a compris que l'urbanisme devait se doter d'une légitimité démocratique » selon le géographe Guy Mercier. «L'urbanisme d'aujourd'hui relève des techniques de régulation de l'opinion publique, ajoute Guy Mercier, car la manière de présenter les projets est de plus en plus importante. Les services d'urbanisme engagent maintenant des spécialistes en relations publiques pour mieux faire passer le message. » (source : [http://www.scom.ulaval.ca/Au.fil.des.evenements/2003/10.02/saintroch.html] consulté le 2 février 2011).

8. Les comptages effectués par des ornithologues semblent confirmer, d'après nos entretiens, le retour de certaines espèces d'oiseaux. En revanche, les hydrobiologistes restent prudents au sujet de la capacité d'accueil de la rivière concernant les espèces de poissons les plus « polluo-sensibles » et les espèces migratrices.

9. Les localités ne manquent pourtant pas d'outils pour "maitriser l'urbanisation" explique un fonctionnaire du ministère chargé des affaires municipales. En 2006, on dénombrait 5296 mises en chantier résidentielles sur le territoire de la Communauté métropolitaine de Québec ( $\mathrm{CMQ}$ ). Ce nombre représente une forte progression par rapport à 2001 où les mises en chantier totales n'étaient que de 2544 . La moyenne annuelle du nombre de mises en chantier entre 2001 et 2006 est de 4973 . Les mises en chantier de maisons individuelles représentaient $61,3 \%$ des mises en chantier totales en 2001. Cette proportion était de $42,5 \%$ en 2006. La construction d'appartements (dont des résidences pour personnes âgées), de jumelés et de maisons en rangées a au contraire fortement progressé de 2001 à 2006 (source: [http://www.cmquebec.qc.ca/territoire/ logement.html], consulté le 17 mai 2011).

10. Québec exige à compter de janvier 2011 des redevances sur l'eau (cf. Règlement sur la redevance exigible pour l'utilisation de l'eau adopté le $1^{\text {er }}$ décembre 2010) (MDDEP, 2010), lesquelles ont immédiatement été dénoncées comme «inefficaces parce que trop peu importantes » par le Parti québécois et la coalition Eau Secours (L.-G. Francœur, Le Devoir, 15 décembre 2010). Le produit des redevances devrait permettre selon le ministère de réaliser plusieurs des engagements gouvernementaux touchant la gestion intégrée des ressources en eau et l'acquisition de connaissances. Par conséquent, la surface financière des organismes de bassin sera peut- être supérieure à ce qu'elle est aujourd'hui d'ici quelques années.

11. Selon plusieurs fonctionnaires interviewés en 2010 à Québec au sujet du rôle du Conseil de Bassin de la rivière Saint-Charles.

12. Des interviewés, parmi lesquels des fonctionnaires municipaux, dénoncent en effet le chevauchement des compétences des organismes non lucratifs ayant les uns et les autres des missions d'utilité publique relatives à la gestion des ressources territoriales. C'est par exemple le cas des commissions régionales des ressources naturelles et du territoire qui relèvent des commissions régionales des élus et des organisations de bassins versants, qui exercent sous la tutelle du Ministère du Développement durable, de l'Environnement et des Parcs. 
13. Excepté Rivière Vivante qui s'est régulièrement invitée dans les débats organisés par la Ville et a influé sur les décideurs pour faire valoir l'intérêt de la destruction des murets de béton (Rivière Vivante, 1996, 1999).

14. Canards Illimités Canada (CIC) est un organisme privé sans but lucratif qui protège et restaure les zones humides. CIC compte 6500 membres au Québec et a aménagé 155 sites. Entretiens avec des ingénieurs en octobre 2009.

15. La charte de la Ville de Québec prévoit cependant que le plan d'urbanisme de la ville peut comprendre un document complémentaire établissant des normes et des critères dont doivent tenir compte les conseils d'arrondissement lorsqu'ils adoptent des règlements d'urbanisme (par exemple un règlement de zonage, un règlement sur les plans d'implantation et d'intégration architecturale). Ces dispositions ont pour but d'accorder au conseil de la ville certains pouvoirs afin d'assurer une harmonisation des règlements d'urbanisme sur l'ensemble municipal. En conséquence, les conseils d'arrondissement doivent prévoir, dans leur règlement, des dispositions au moins aussi contraignantes que les conditions établies dans le do-cument complémentaire de la ville. Cf. Charte de la Ville de Québec (LRQ., c.C-11.5) article 72.2.

16. La Communauté métropolitaine de Québec $(\mathrm{CMQ})$ devait adopter son premier schéma au plus tard le 31 décembre 2006. Le territoire de la $\mathrm{CMQ}$ correspond à peu près à celui de la région métropolitaine de recensement (RMR). $\mathrm{LaCMQ}$ regroupe les territoires des villes de Lévis et de Québec et les territoires des MRC de la Jacques-Cartier, de La Côte-deBeaupré et de l'île d'Orléans. Il couvre une superficie de 3 066,3 kilomètres carrés dont $27,5 \%$ sont situés en zone agricole, désignée en vertu de la Loi sur la protection du territoire et des activités agricoles. Sa population est de 687207 habitants. Deux des 26 municipalités qu'elle regroupe représentent $92 \%$ de la population métropolitaine totale. Il s'agit de Québec (507 986) et de Lévis (121 999).

17. À l'instar du schéma d'aménagement et de développement, le plan d'urbanisme n'a pas d'effet juridique direct sur le citoyen et, de ce fait, les dispositions du plan ne peuvent à elles seules servir de justification pour refuser la délivrance d'un permis de construction ou de lotissement. Pour justifier un tel refus, les intentions qui y sont exprimées doivent être mises en application dans les règlements d'urbanisme (source : [ http://www.mamrot.gouv.qc.ca/amenagement/outils/amen_outi_plan_plan.asp] consulté le 27 avril 2010).

18. Source : communiqué de la communauté urbaine de Québec relatif à la protection de la ressource en eau en date du 10 novembre 2010.

\section{RÉSUMÉS}

La « renaturation » de la section aval de la rivière Saint-Charles à Québec (1996-2009) marque un tournant en matière de politique de l'eau au plan municipal. Cette « renaturation » a en effet été précédée par des opérations de remblaiement (années 1960), de bétonnage des berges et de réduction des effets des marées (années 1970) menées à l'époque dans une perspective hygiéniste et esthétique. Au cours des années 1990, la Ville de Québec a constaté que ces interventions ont 
profondément perturbé le fonctionnement de l'écosystème aquatique et n'ont pas eu l'effet escompté sur les utilisateurs potentiels qui s'en détournaient toujours. La Ville de Québec a alors amorcé une politique de restauration spectaculaire de la basse Saint-Charles qui lui a permis de lier la reconquête de la rivière à la valorisation commerciale et récréative des parcelles riveraines du cours d'eau, faisant ainsi converger les demandes des riverains, les exigences l'Administration et les aspirations des élus. À l'échelle métropolitaine cependant, l'étalement urbain constitue une menace sérieuse sur la ressource en eau car il risque de masquer les efforts consentis en aval par la Ville. L'article montre que la municipalité, perçue comme le seul acteur légitime aux yeux des aménageurs, peine à prendre en compte la protection de la ressource en eau dans le cadre des règlements et schémas d'urbanisme à l'échelle du bassin versant comme l'y incite la Politique nationale de l'eau du Québec de 2002.

Since the 1960s the Saint-Charles River has been the object of many developments. In particular, the banks of the River have been consolidated and riparian wetlands have been backfilled. A dam was built to counter the effects of the tides. The objective of these different development was to improve the quality of water and attract users. From the 1990s, municipal authority noted the failure of the developments made in the 1960s? It was decided to « it » River in the urban portion to meet seemingly contradictory requirements of developers, users, ecologists and residents. «Renaturation» of the Saint-Charles River in Quebec (1996-2009) is a milestone for the city of Quebec. Indeed, this operation of renewing urban led by the city is a success. Unfortunately this spectacular urban operation concerns only a small portion of the watershed. The challenge for the city of Quebec is now limiting urban sprawl.

\section{INDEX}

Mots-clés : gouvernance, renaturation, pollution, politique de l'eau, projet urbain

Keywords : urban project, renaturation, pollution, water policy governance

Index géographique : Québec, Saint-Charles

\section{AUTEUR}

\section{ALEXANDRE BRUN}

Acteurs-Ressources-Territoires dans le Développement - UMR 5281 CNRS (Université Paul Valéry Montpellier 3), Route de Mende, 34199 Montpellier Cedex 05, France abrungeographe@yahoo.fr 